\title{
Hybrid Block Method Algorithms for Solution of First Order Initial Value Problems in Ordinary Differential Equations
}

\section{Ajileye $\mathbf{G}^{*}$, Amoo SA and Ogwumu OD}

Department of Mathematics and Statistics, Federal University Wukari, Wukari, Taraba State, Nigeria

\begin{abstract}
In this paper, we consider the derivation of hybrid block method for the solution of general first order Initial Value Problem (IVP) in Ordinary Differential Equation. We adopted the method of Collocation and Interpolation of power series approximation to generate the continuous formula. The properties and feature of the method are analyzed and some numerical examples are also presented to illustrate the accuracy and effectiveness of the method.
\end{abstract}

Keywords: Collocation; Interpolation; Linear multistep method; Hybrid and power series polynomial

\section{Introduction}

In recent times, the integration of Ordinary Differential Equations (ODEs) is carried out using some kinds of block methods. In this paper, we propose an order six block integrator for the solution of first-order ODEs of the form:

$$
y^{\prime}=f(x, y), y(a)=y_{o}, \quad x \in[a . b]
$$

Where $f$ is continuous within the interval of integration $[\mathrm{a}, \mathrm{b}]$. We assume that $f$ satisfies Lipchitz condition which guarantees the existence and uniqueness of solution of eqn. (1). For the discrete solution of (1) by linear multi-step method has being studied by authors like [1] and continuous solution of eqn. (1) and [2-4]. One important advantage of the continuous over discrete approach is the ability to provide discrete schemes for simultaneous integration. These discrete schemes can be reformulated as general linear methods (GLM) [5]. The block methods are self-starting and can be applied to both stiff and non-stiff initial value problem in differential equations. More recently, authors like [6$10]$ and to mention few, these authors proposed methods ranging from predictor- corrector to hybrid block method for initial value problem in ordinary differential equation.

In this work, hybrid blocks method with two off-grid using Power series expansion [11,12]. This would help in coming up with a more computationally reliable integrator that could solve first order differential equations problems of the form eqn. (1).

\section{Derivation of Hybrid Method}

In this section, we intend to construct the proposed two-step $L M M s$ which will be used to generate the method. We consider the power series polynomial of the form:

$$
P(x)=\sum_{j=0}^{n} a_{j} x^{j}
$$

which is used as our basis to produce an approximate solution to (1.0) as

$$
y(x)=\sum_{j=0}^{m+t-1} a_{j} x^{j}
$$

And

$$
y^{\prime}(x)=\sum_{j=0}^{m+t-1} j a_{j} x^{j-1}=f(x, y)
$$

where $\mathrm{a}_{\mathrm{i}}$ are the parameters to be determined, $\mathrm{m}$ and $\mathrm{t}$ are the points of collocation and interpolation respectively. This process leads to $(\mathrm{m}+\mathrm{t}-1)$ of non-linear system of equations with $(\mathrm{m}+\mathrm{t}-1)$ unknown coefficients, which are to be determined by the use of Maple 17 Mathematical software.

\section{Hybrid Block Method}

Using eqns. (3) and (4), $m=1$ and $t=5$ our choice of degree of polynomial is $(m+t-1)$. Eqn. (3) is interpolates at the point $x=x_{n}$ and eqn. (4) is collocated at $x=\left(0, \frac{1}{2}, 1, \frac{3}{2}, 2\right)$ which lead to system of
equation of the form

$$
\begin{aligned}
& \sum_{j=0}^{m+t-1} a_{j} x_{n+i}^{j}=y_{n+i} \quad i=0 \\
& \sum_{j=0}^{m+t-1} j a_{j} x_{n+i}^{j}=f_{n+i} \quad i=\left(0, \frac{1}{2}, 1 \frac{3}{2}, 2\right)
\end{aligned}
$$

With the mathematical software, we obtain the continuous formulation of eqns. (5) and (6) of the form

$$
y(x)=\alpha_{0} y_{n}+h\left[\beta_{0} f_{n}+\beta_{\frac{1}{2}} f_{n+\frac{1}{2}}+\beta_{1} f_{n+1}+\beta_{\frac{3}{2}} f_{n+\frac{3}{2}}+\beta_{2} f_{n+2}\right]
$$

After obtaining the values of $\alpha_{j}$ and $\beta_{j} j=0$ and $i=\left(0, \frac{1}{2}, 1 \frac{3}{2}, 2\right)$ in eqn. (7)

We evaluated at the point $x=x_{n+j} j=\left(1, \frac{1}{2}, \frac{3}{2}, 2\right)$ which gives the following set of discrete schemes to form our hybrid block method.

*Corresponding author: Ajileye G, Department of Mathematics and Statistics,
Federal University Wukari, Wukari, Taraba State, Nigeria, Tel: 2348034906427; E-mail: ajisco4live@yahoo.com

Received April 16, 2018; Accepted May 30, 2018; Published June 19, 2018

Citation: Ajileye G, Amoo SA, Ogwumu OD (2018) Hybrid Block Method Algorithms for Solution of First Order Initial Value Problems in Ordinary Differential Equations. J Appl Computat Math 7: 390. doi: 10.4172/2168-9679.1000390

Copyright: @ 2018 Ajileye G, et al. This is an open-access article distributed under the terms of the Creative Commons Attribution License, which permits unrestricted use, distribution, and reproduction in any medium, provided the original author and source are credited. 


$$
\begin{aligned}
& y_{n+1}=y_{n}+\frac{29}{180} h f_{n}+\frac{31}{45} h f_{n+1 / 2}+\frac{2}{15} h f_{n+1}+\frac{1}{45} h f_{n+3 / 2}-\frac{1}{180} h f_{n+2} \\
& y_{n+1 / 2}=y_{n}+\frac{251}{1440} h f_{n}+\frac{323}{720} h f_{n+1 / 2}-\frac{11}{60} h f_{n+1}+\frac{53}{720} h f_{n+3 / 2}-\frac{19}{1440} h f_{n+2} \\
& y_{n+3 / 2}=y_{n}+\frac{27}{160} h f_{n}+\frac{51}{80} h f_{n+1 / 2}+\frac{9}{20} h f_{n+1}+\frac{21}{80} h f_{n+3 / 2}-\frac{3}{160} h f_{n+2} \\
& y_{n+2}=y_{n}+\frac{7}{45} h f_{n}+\frac{32}{45} h f_{n+1 / 2}+\frac{4}{15} h f_{n+1}+\frac{32}{45} h f_{n+3 / 2}+\frac{7}{45} h f_{n+2}
\end{aligned}
$$

Eqn. (8.0) are of uniform order 5, with error constant as follows

$$
\left[\frac{1}{5760}, \frac{3}{10240}, \frac{3}{10240},-\frac{1}{15120}\right]^{T} \text {. }
$$

\section{Consistency}

Definition: The Linear Multistep method is said to be consistent if it is of order

$\mathrm{P} \geq 1$ and its first and second characteristic polynomial defined as $\rho(z)=\sum_{j=0}^{k} \alpha_{j} z^{j}$ and $\sigma(z)=\sum_{j=0}^{k} \beta_{j} z^{j}$ where $\mathrm{Z}$ satisfies (i) $\sum_{j=0}^{k} \alpha_{j}=0,(i i) \rho^{\prime}(1)=0,(i i i) \rho^{\prime \prime}(1)=2 ! \sigma(1)$, See Lambart (1973).

The discrete Schemes derived are all of order than one and satisfy the condition (i)-(iii).

\section{Zero Stability of the block Method}

The block method is defined by Fatunla (1988) as

$$
\begin{aligned}
& Y_{m}=\sum_{i=0}^{k} A_{i}+h \sum_{i=0}^{k} B_{i} F_{m-i} \\
& \text { where } Y_{m}=\left[y_{n}, y_{n+1}, y_{n+2}, \ldots, y_{n+r-1}\right]^{T} \\
& F_{m}=\left[f_{n}, f_{n+1}, f_{n+2}, \ldots, f_{n+r-1}\right]^{T}
\end{aligned}
$$

$A_{i}^{\prime} s$ and $B_{i}^{\prime} s$ are chosen $\mathrm{r} \times \mathrm{r}$ matrix coefficient and $\mathrm{m}=0,1,2 \ldots$ represents the block number, $n=m \gamma$ the first step number in the $m$-th block and $\mathrm{r}$ is the proposed block size.

The block method is said to be zero stable if the roots of $R_{j} j=1(1) k$ of the first characteristics polynomial is

$$
\rho(R)=\operatorname{det}\left[\sum_{i=0}^{k} A_{i} R^{k-1}\right]=0, A_{0}=I
$$

satisfies $\left|\mathbf{R}_{\mathrm{j}}\right| \leq \mathbf{1}$, if one of the roots is +1 , then the root is called Principal Root of $\rho(R)$.

$$
\begin{aligned}
& {\left[\begin{array}{llll}
1 & 0 & 0 & 0 \\
0 & 1 & 0 & 0 \\
0 & 0 & 1 & 0 \\
0 & 0 & 0 & 1
\end{array}\right]\left[\begin{array}{c}
y_{n+1} \\
y_{n+1 / 2} \\
y_{n+3 / 2} \\
y_{n+2}
\end{array}\right]=\left[\begin{array}{llll}
0 & 0 & 0 & 1 \\
0 & 0 & 0 & 1 \\
0 & 0 & 0 & 1 \\
0 & 0 & 0 & 1
\end{array}\right]\left[\begin{array}{c}
y_{n-3 / 2} \\
y_{n-1 / 2} \\
y_{n-1} \\
y_{n}
\end{array}\right]+h\left[\begin{array}{cccc}
\frac{31}{45} & \frac{2}{15} & \frac{1}{45} & \frac{1}{180} \\
\frac{323}{720} & \frac{-11}{60} & \frac{53}{720} & \frac{-19}{1440} \\
\frac{51}{80} & \frac{9}{20} & \frac{21}{80} & \frac{-3}{160} \\
\frac{32}{45} & \frac{4}{15} & \frac{32}{45} & \frac{7}{45}
\end{array}\right]\left[\begin{array}{c}
f_{n+1 / 2} \\
f_{n+1} \\
f_{n+3 / 2} \\
f_{n+2}
\end{array}\right]} \\
& +\left[\begin{array}{llll}
0 & 0 & 0 & \frac{29}{180} \\
0 & 0 & 0 & \frac{251}{1440} \\
0 & 0 & 0 & \frac{27}{160} \\
0 & 0 & 0 & \frac{7}{45}
\end{array}\right]\left[\begin{array}{c}
f_{n-3 / 2} \\
f_{n-1} \\
f_{n-1 / 2} \\
f_{n}
\end{array}\right]
\end{aligned}
$$

Where

$$
A^{(0)}=\left[\begin{array}{llll}
1 & 0 & 0 & 0 \\
0 & 1 & 0 & 0 \\
0 & 0 & 1 & 0 \\
0 & 0 & 0 & 1
\end{array}\right], A^{(1)}=\left[\begin{array}{llll}
0 & 0 & 0 & 1 \\
0 & 0 & 0 & 1 \\
0 & 0 & 0 & 1 \\
0 & 0 & 0 & 1
\end{array}\right], B^{(0)}=\left[\begin{array}{cccc}
\frac{31}{45} & \frac{2}{15} & \frac{1}{45} & \frac{1}{180} \\
\frac{323}{720} & \frac{-11}{60} & \frac{53}{720} & \frac{-19}{1440} \\
\frac{51}{80} & \frac{9}{20} & \frac{21}{80} & \frac{-3}{160} \\
\frac{32}{45} & \frac{4}{15} & \frac{32}{45} & \frac{7}{45}
\end{array}\right]
$$

and

$$
B^{(0)}=\left[\begin{array}{cccc}
0 & 0 & 0 & \frac{29}{180} \\
0 & 0 & 0 & \frac{251}{1440} \\
0 & 0 & 0 & \frac{27}{160} \\
0 & 0 & 0 & \frac{7}{45}
\end{array}\right]
$$

The first characteristics polynomial of the scheme is

$$
\begin{aligned}
& \rho(\lambda)=\operatorname{det}\left[\lambda A^{0}-A^{1}\right] \\
& \rho(\lambda)=\operatorname{det}\left[\begin{array}{cccc}
\lambda & 0 & 0 & -1 \\
0 & \lambda & 0 & -1 \\
0 & 0 & \lambda & -1 \\
0 & 0 & 0 & \lambda-1
\end{array}\right] \\
& \left|\begin{array}{cccc}
\lambda & 0 & 0 & -1 \\
0 & \lambda & 0 & -1 \\
0 & 0 & \lambda & -1 \\
0 & 0 & 0 & \lambda-1
\end{array}\right|=0 \\
& \lambda^{3}(\lambda-1)=0 \\
& \lambda_{1}=\lambda_{2}=\lambda_{3}=0 \text { or } \lambda_{4}=1
\end{aligned}
$$$$
\rho(\lambda)=\operatorname{det}\left[\left(\begin{array}{llll}
\lambda & 0 & 0 & 0 \\
0 & \lambda & 0 & 0 \\
0 & 0 & \lambda & 0 \\
0 & 0 & 0 & \lambda
\end{array}\right)-\left(\begin{array}{llll}
0 & 0 & 0 & 1 \\
0 & 0 & 0 & 1 \\
0 & 0 & 0 & 1 \\
0 & 0 & 0 & 1
\end{array}\right)\right]
$$

We can see clearly that no root has modulus greater than one (i.e $\left.\left.\lambda_{\mathrm{i}} \leq 1\right)\right) \forall i$. The hybrid block method is zero stable.

\section{Numerical Examples}

\section{Problem 1}

$y^{\prime}=y, \quad y(0)=1, h=0.1$

\section{Exact solution}

$\mathrm{y}(\mathrm{x})=\exp (\mathrm{x})($ Table 1$)$.

\section{Problem 2}

$y^{\prime}=0.5(1-y), \quad y(0)=0.5, h=0.1$

\section{Exact solution}

$y(x)=1-0.5 e-0.5 x($ Table 2$)$.

\section{Discussion of Result}

We observed that from the two problems tested with this proposed 
Citation: Ajileye G, Amoo SA, Ogwumu OD (2018) Hybrid Block Method Algorithms for Solution of First Order Initial Value Problems in Ordinary Differential Equations. J Appl Computat Math 7: 390. doi: 10.4172/2168-9679.1000390

Page 3 of 3

\begin{tabular}{|c|c|c|c|c|}
\hline $\mathbf{x}$ & Exact Solution & Scheme & Error in Scheme & Error [2] \\
\hline 0.1 & 1.105170918075648 & 1.105170917860730 & $2.149179 \mathrm{E}-10$ & $1.226221039551945 \mathrm{e}-05$ \\
\hline 0.2 & 1.221402758160170 & 1.221402757685120 & $4.7505 \mathrm{E}-10$ & $1.355183832019158 \mathrm{e}-05$ \\
\hline 0.3 & 1.349858807576003 & 1.349858806788490 & 7.875129E-10 & $1.497709759790133 e-05$ \\
\hline 0.4 & 1.491824697641270 & 1.491824696480820 & 1.16045E-09 & $1.655225270247307 \mathrm{e}-05$ \\
\hline 0.5 & 1.648721270700128 & 1.648721269097010 & 1.603118E-09 & $1.829306831546695 \mathrm{e}-05$ \\
\hline 0.6 & 1.822118800390509 & 1.822118798264440 & 2.126069E-09 & $2.021696710463594 \mathrm{e}-05$ \\
\hline 0.7 & 2.013752707470477 & 2.013752704729200 & 2.741277E-09 & $2.234320409577606 \mathrm{e}-05$ \\
\hline 0.8 & 2.225540928492468 & 2.225540925030090 & 5.989459E-09 & $2.469305938346267 \mathrm{e}-05$ \\
\hline 0.9 & 2.459603111156950 & 2.459603106852120 & 4.30483-09 & $2.729005110868599 \mathrm{e}-05$ \\
\hline 1.0 & 2.718281828459046 & 2.718281824122030 & 4.337016E-09 & $3.01601708376864 \mathrm{e}-05$ \\
\hline
\end{tabular}

Table 1: Comparison of approximate solution of problem 1.

\begin{tabular}{|c|c|c|c|c|}
\hline $\mathbf{x}$ & Exact Solution & Scheme & Error in Scheme & Error [7] \\
\hline 0.1 & 0.524385287749643 & 0.524385287750861 & 1.218026E-13 & $5.574430 \mathrm{e}-012$ \\
\hline 0.2 & 0.547581290982020 & 0.547581290981880 & $1.399991 \mathrm{E}-13$ & $3.946177 e-012$ \\
\hline 0.3 & 0.569646011787471 & 0.569646011786286 & $1.184941 \mathrm{E}-12$ & $8.183232 e-012$ \\
\hline 0.4 & 0.590634623461009 & 0.590634623462548 & $1.538991 \mathrm{E}-12$ & $3.436118 e-011$ \\
\hline 0.5 & 0.610599608464297 & 0.610599608463187 & $1.110001 \mathrm{E}-12$ & $1.929743 e-010$ \\
\hline 0.6 & 0.629590889659141 & 0.629590889658614 & $5.270229 \mathrm{E}-12$ & $1.879040 \mathrm{e}-010$ \\
\hline 0.7 & 0.647655955140643 & 0.647655955142752 & $2.10898 \mathrm{E}-12$ & $1.776835 \mathrm{e}-010$ \\
\hline 0.8 & 0.664839976982180 & 0.664839976969201 & 1.297895E-11 & $1.724676 \mathrm{e}-010$ \\
\hline 0.9 & 0.681185924189113 & 0.681185924158290 & 3.08229E-11 & $1.847545 e-010$ \\
\hline 1.0 & 0.696734670143683 & 0.696734670139561 & 4.121925E-11 & $3.005770 \mathrm{e}-010$ \\
\hline
\end{tabular}

Table 2: Comparison of approximate solution of problem 2.

block hybrid method the results converges to exact solutions and also compared favorably with the existing similar methods (see Tables 1 and 2).

\section{Conclusion}

In this paper, we have presented Hybrid block method algorithm for the solution of first order ordinary differential equations. The approximate solution adopted in this research produced a block method with stability region. This made it to perform well on problems. The block method proposed was found to be zero-stable, consistent and convergent.

\section{References}

1. Lambert JD (1973) Computational methods in ordinary differential equations.

2. SO A, Ibijola EA (2015) A new numerical method for solving first order differential equations. Am J Appl Math Stat 3: 156-160.

3. Onumanyi P, Awoyemi DO, Jator SN, Sirisena UW (1994) New linear mutlistep methods with continuous coefficients for first order initial value problems. J Nig Math Soc 13: 37-51.

4. Onumanyi $P$, Sirisena UW, Jator SN (1999) Continuous finite difference approximations for solving differential equations. Int J of Comp Math 72: 15-27.
5. Butcher JC (1993) General linear methods for the parallel solution of ordinary differential equations. In Contributions In Numerical Mathematics, pp: 99-111.

6. James AA, Adesanya AO, Sunday J (2012) Uniform Order Continuous Block Hybrid Method for the Solution of First Order Ordinary Differential Equations. IOSR J Math 2278: 08.

7. Sunday J, Odekunle MR, Adesanya AO (2013) Order six block integrator for the solution of first-order ordinary differential equations. Int J Math soft comp 3: 87-96.

8. Mohammed U, Yahaya YA (2010) Fully implicit four point block backward difference formula for solving first-order initial value problems. Leonardo Journal of Sciences 16: 21-30.

9. Skwame Y, Sunday J, Ibijola EA (2012) L-stable block hybrid simpson's methods for numerical solution of initial value problems in stiff ordinary differential equations. Int J Pure Appl Sci Technol 11: 45-54.

10. Donald JZ (2009) Construction of two steps Simpson's multistep method as parallel integrator for the solution of ordinary differential equation. Bagale $J$ Pure Appl Sci 7: 1-12.

11. Awoyemi DO, Ademiluyi RA, Amuseghan E (2008) Off-grid points exploitation in the development of more accurate collocation method for solving ODEs. Journal of the Nigerian Association of Mathematical Physics 12.

12. Dahlquist G (1956) Convergence and stability in the numerical integration of ordinary differential equations. Mathematica Scandinavica 33-53. 